\title{
The construction of recombinant Lactobacillus casei vaccine of PEDV and its immune responses in mice
}

\author{
Xiaowen $\mathrm{Li}^{1,2,3 \dagger}$, Bingzhou Zhang ${ }^{1,2+}$, Dasheng Zhang ${ }^{4}$, Sidang Liu $^{3^{*}}$ and Jing Ren ${ }^{2,5^{*}}$
}

\begin{abstract}
Background: Porcine epidemic diarrhea (PED) is a contagious intestinal disease caused by porcine epidemic diarrhea virus (PEDV) characterized by vomiting, diarrhea, anorexia, and dehydration, which have caused huge economic losses around the world. At present, vaccine immunity is still the most effective method to control the spread of PED. In this study, we have constructed a novel recombinant L. casei-OMP16-PEDVS strain expressing PEDVS protein of PEDV and OMP16 protein of Brucella abortus strain. To know the immunogenicity of the recombinant L. casei-OMP16-PEDVS candidate vaccine, it was compared with BL21-OMP16-PEDVS-F, BL21-OMP16PEDVS, and BL21-PEDVS recombinant protein.

Results: The results showed that we could detect higher levels of IgG, neutralizing antibody, IL-4, IL-10, and INF- $\gamma$ in serum and IgA in feces of L. casei-OMP16-PEDVS immunized mice, which indicated that L. casei-OMP16-PEDVS candidate vaccine could induce higher levels of humoral immunity, cellular immunity, and mucosal immunity.

Conclusion: Therefore, L. casei-OMP16-PEDVS is a promising candidate vaccine for prophylaxis of PEDV infection.
\end{abstract}

Keywords: PEDV, Lactobacillus casei vaccine, PEDV S protein, OMP16, Immune responses

\section{Introduction}

Porcine epidemic diarrhea (PED) is caused by porcine epidemic diarrhea virus (PEDV) with symptoms including diarrhea, vomiting, anorexia, dehydration, and weight loss in piglets $[1,2]$. Pigs of all ages can be infected with different symptoms and the mortality rate in piglets is up to $100 \%$ [3], which have led to huge economic losses all around the world. To control the spread of PEDV, most kinds of vaccines are constructed, such as aluminumhydroxide-adjuvanted inactivated vaccine, bivalent inactivated Transmissible Gastroenteritis Virus (TGEV) and

\footnotetext{
* Correspondence: liusid@sdau.edu.cn; renjing879@163.com

${ }^{+}$Xiaowen Li and Bingzhou Zhang are co-first author and contributed equally to this article

${ }^{3}$ College of Animal Science and Technology, Shandong Agricultural University, Tai'an, China

${ }^{2}$ Shandong Swine Herd Health Big Data and Intelligent Monitoring Engineering Laboratory, Tai'an, China

Full list of author information is available at the end of the article
}

PEDV vaccine, and attenuated PEDV vaccine [4]. Although these vaccines play an important role in controlling PED, they all have their defects. Inactivated vaccines cannot activate cellular immune responses, attenuated vaccine is not very safe, and they all cannot induce sufficient production of virus-specific IgA antibodies of mucosal immune responses. Therefore, it is necessary and urgent to develop a new vaccine to control PED.

Lactobacillus casei is often considered to be a kind of safe vector system for targeted delivery of antigens in oral immunization, with beneficial effects on the health of humans and animals [5]. Meanwhile, it can be used as a delivery system to regulate the T-helper cell response and stimulate the secretion of specific IgAs for mucosal immunity [6]. On the other hand, Lactobacillus casei recombinant vaccine is easier administration, lesser chance of hypersensitivity reaction, and more costeffective compared with traditional vaccines. Based on 
the reports, Lactobacillus casei recombinant vaccines have been successfully used in the prevention and control of human papillomavirus, Streptococcus pneumonia, and Escherichia coli [7-9]. There are also some similar attempts in designing of PED vaccines. The researches find that a recombinant Lactococcus lactis strain expressing a variant porcine epidemic diarrhea virus S1 gene could induce high levels of IL-4 and IFN- $\gamma$ in immunized mice [10]. Lactobacillus casei-based anti-PEDV vaccine expressing microfold cell-targeting peptide Co1 fused with the COE antigen of PEDV could also induce effective immune response [11]. To improve the effectiveness of PEDV vaccine. In this study, we construct a new Lactobacillus casei recombinant vaccine of PED, which can stimulate stronger mucosal, humoural and cellular immune responses against PEDV infection via oral administration.

PEDV, a member of the coronaviridae family, consisted by four structural proteins which contain the 150-220 kDa glycosylated spike (S) protein, the 20-30 $\mathrm{kDa}$ membrane $(\mathrm{M})$ protein, the $7 \mathrm{kDa}$ envelope (E) protein, and $58 \mathrm{kDa}$ nucleocapsid $(\mathrm{N})$ protein [12]. Thereinto, the S protein can be divided into S1 (1-735 amino acid) and S2 (736-last amino acid) domains [13], and S1 protein includes the receptor-binding region and the main neutralizing epitopes [14]. Vaccine adjuvant acts as an immunomodulator can induce and enhance immune responses against co-delivered antigens. OMP16 protein of Brucella abortus strain was verified that could activate dendritic cells in vivo, induces a th1 immune response, and was a promising self-adjuvanting vaccine [15-17]. Therefore, OMP16 protein was inserted into the Lactobacillus casei recombinant vaccine in our study.

So far, few studies about the Lactobacillus casei recombinant vaccine of PEDV are reported. Therefore, this study is aimed to construct a novel Lactobacillus casei candidate oral vaccine, which can supply better humoral immunity, cellular immunity, and mucosal immunity to prevent the spread of PED.

\section{Materials and methods}

Bacterial strains, viruses, culture conditions, plasmids, and primers

The bacterial strains, plasmids, and primers used in this study are listed in Table 1. The standard reference strain of Lactobacillus casei ATCC 393 was cultured in de Man Rogosa and Sharpe (MRS) broth at $37^{\circ} \mathrm{C}$ [18]. The BL21 (DE3) and DH5 $\alpha$ were cultured in Luria-Bertani (LB) medium at $37^{\circ} \mathrm{C}$ [19]. The recombinant Lactobacillus casei, BL21 (DE3), and DH5 $\alpha$ strains were cultured in the corresponding medium with proper antibiotics, respectively. Brucella abortus was grown in Tryptic Soy Broth (TSB) or Tryptic Soy Agar (TSA) medium (Difco Laboratories, Detroit, MI, USA) at $37^{\circ} \mathrm{C}$. The Vero cells infected with PEDV strains were cultured in DMEM (Gibco, Langley, VA, USA) supplemented with $10 \mu \mathrm{g} / \mathrm{mL}$

Table 1 Characteristics of bacterial strains, plasmids, and primers used in this study

\begin{tabular}{|c|c|c|}
\hline Strain/plasmid/primer & Characteristics and/or sequences & Source/reference \\
\hline \multicolumn{3}{|l|}{ Strain } \\
\hline $\mathrm{DH} 5 \mathrm{a}$ & Genotype: supE44 $\Delta$ lacU169 ( $(80 \mathrm{lacZ} \Delta \mathrm{M} 15)$ hsdR17 recA1 endA1 gyrA96 thi-1 relA1 & TaKaRa (Otsu, Japan) \\
\hline BL21 & Genotype: F-ompT hsdS (rB-mB) gal dcm (DE3) & TaKaRa (Otsu, Japan) \\
\hline Lactobacillus casei & ATCC393, used as a vector system for targeted delivery of antigens in oral immunization. & Preserved in our lab \\
\hline Brucella abortus & Brucella abortus S19, supply gene sequence of omp16 gene & Preserved in our lab \\
\hline PEDV & AJ1 102, variant strain, isolated in China. & Preserved in our lab \\
\hline \multicolumn{3}{|l|}{ Plasmids } \\
\hline pET28a $(+)$ & The expression vector of Escherichia coli, Kan ${ }^{r}$ & Preserved in our lab \\
\hline pVE5523 & The expression vector of Lactobacillus casei, Amp ${ }^{r}$ & BioVector NTCC \\
\hline pET28a-PEDVS & The recombinant vector of pET28a and S protein of PEDV, Kan ${ }^{r}$ & This study \\
\hline pET28a-OMP16-PEDVS & $\begin{array}{l}\text { The recombinant vector of pET28a and fusion protein (S protein of PEDV and OMP16 } \\
\text { protein of Brucella abortus), Kanr }\end{array}$ & This study \\
\hline pVE5523-OMP16-PEDVS & $\begin{array}{l}\text { The recombinant vector of pVE5523 and fusion protein (S protein of PEDV and OMP16 } \\
\text { protein of Brucella abortus), Ampr }\end{array}$ & This study \\
\hline \multicolumn{3}{|l|}{ Primers } \\
\hline PEDVS-F1/R1 & $\begin{array}{l}\text { F1: CCGGAATTCATGCTGAGTCATGAACAGCC } \\
\text { R1: TGCTCTAGATTAATATGCAGCCTGCTCTG }\end{array}$ & This study \\
\hline PEDVS-F2/R2 & $\begin{array}{l}\text { F2:GGTGGTGGCGGTAGCGGCGGTGGTGGCTCTGGTGGCGGCGGTTCTTTCTTTGTTACTTTGCCAT } \\
\text { R2: CGCGATATCTTAATATGCAGCCTGCTCTG }\end{array}$ & This study \\
\hline OMP16-F/R & $\begin{array}{l}\text { F: CGCGTCGACATGGCGTCAAAGAAGAACCTTCCG } \\
\text { R:AGAACCGCCGCCACCAGAGCCACCACCGCCGCTACCGCCACCACCGGTACCCCGTCCGGCCCCGT }\end{array}$ & This study \\
\hline
\end{tabular}


trypsin (Gibco, Langley, VA, USA) [20]. The pVE5523 and pET28a $(+)$ plasmids were expression vectors of Lactobacillus casei and Escherichia coli, respectively.

\section{The construction of recombinant Lactobacillus casei and BL21 strains}

The recombinant expression plasmids were constructed based on the plasmids and primers in Table 1. At first, the partial sequence of PEDV $S$ gene (493-708 amino acid), the partial sequence of PEDV S' gene (493-708 amino acid), and the partial sequence of Brucella abortus OMP16 gene (26-168 amino acid) were amplified using primer pairs PEDVS-F1/R1, PEDVS-F2/R2, and OMP16-F/R, respectively. Subsequently, the overlap extension method was used in OMP16 and PEDVS' fragments to construct a new fragment OMP16-PEDVS with linker polypeptide (GGGGSGGGGSGGGGS) stuck in the middle. Then, the fragments PEDVS was inserted into pET28a plasmid with EcoRI/XbaI restriction enzymes to generate recombinant plasmid pET28a-PEDVS, and the new fragments OMP16-PEDVS was inserted into pET28a and pVE5523 plasmids with EcoRI/XbaI and SalI/EcoRV restriction enzymes to generate recombinant plasmid pET28a-OMP16-PEDVS and pVE5523-OMP16-PEDVS, respectively. The recombinant plasmids (pET28a-PEDVS, pET28a-OMP16-PEDVS, and pVE5523-OMP16-PEDVS) were transformed into BL21 (DE3) or Lactobacillus casei ATCC393 by transformation or electroporation based on the reported paper [21].

\section{Analysis of protein expression by western blot}

The protein expression of BL21-pET28a-PEDVS, BL21pET28a-OMP16-PEDVS, and L. casei-pVE5523-OMP16PEDVS strains were detected based on the reported method with some modification [21-23]. The recombinant strains BL21-pET28a-PEDVS and BL21-pET28aOMP16-PEDVS were cultured in LB broth and IPTG was used to harvest to pET28a-PEDVS and pET28a-OMP16PEDVS proteins. The blank vector was used as a negative control. After cell lysis and centrifugation, the supernatant and sediment were collected. Then, the target proteins were purified using the Nickel affinity chromatography column based on the previous study [24]. Meanwhile, the cultural supernatant of the recombinant L. caseipVE5523-OMP16-PEDVS strain was also harvested by centrifugation at $9000 \times \mathrm{g}$ for $10 \mathrm{~min}$ at $4{ }^{\circ} \mathrm{C}$. Whereafter, the samples with sodium dodecyl sulfate (SDS) loading buffer were boiled 10 mins. The proteins were separated by $12 \%$ sodium dodecyl sulfate-polyacrylamide gel electrophoresis (SDS-PAGE) and then transferred into PVDF membranes (Millipore, Mississauga, ON, Canada). Membranes were blocked with 5\% skimmed milk for $2 \mathrm{~h}$ at $37^{\circ} \mathrm{C}$ and then incubated with murine monoclonal antibody of $\mathrm{S}$ protein overnight at $4{ }^{\circ} \mathrm{C}$ and HRP conjugated goat anti-mouse IgG (ABclonal, Wuhan, China) for $2 \mathrm{~h}$ at $37^{\circ} \mathrm{C}$. The protein bands were visualized using the Clarity $^{\text {min }}$ Western ECL Blotting Substrate (Bio-Rad, Hercules, CA, USA).

\section{Immunization and sample collection}

The immunogenicity of recombinant Lactobacillus casei vaccine was evaluated using six-week-old female specific pathogen-free (SPF) BALB/c mice $[10,18]$, which were purchased from Shandong Agricultural University animal center. A total of 50 mice were randomly divided into 5 groups with 10 mice in each group. The mice were immuned with recombinant Lactobacillus casei vaccine and purified protein (pET28a-PEDVS, pET28aOMP16-PEDVS, pET28a-OMP16-PEDVS+Freund's complete adjuvant), respectively. The immunization protocol was performed based on Table 2 and a booster immunization was given after 13 days.

For the serological study, serum was collected on 0 , 14 , and 28 days post-immunization (dpi) via tail vein punching and stored at $-20^{\circ} \mathrm{C}$ until use. Feces were collected 1 day before vaccination and every boosting time. For IgA detection, feces were diluted (w/v) with $0.05 \mathrm{M}$ sodium EDTA at 1:4 ratios just after collection and incubated for $14 \mathrm{~h}$ at $4{ }^{\circ} \mathrm{C}$ following proper mixing. The supernatant was collected by $12,000 \times \mathrm{g}$ centrifugation and preserved at $-20^{\circ} \mathrm{C}$ until use. At 28th dpi; three mice from each group were sacrificed and the intestine was processed for IgA detection according to the author described previously $[10,18,23]$.

\section{Determination analysis of antibody levels}

The levels of IgG in the sera and IgA in the feces were measured by the ELISA methods with some modification $[18,23]$. The methods were as follows: Polystyrene microliter plates were coated overnight at $4{ }^{\circ} \mathrm{C}$ with $100 \mu \mathrm{L} 10 \mu \mathrm{g} / \mathrm{mL}$ PEDVS protein, OMP16-PEDVS protein, or $100 \mu \mathrm{L}$ recombinant L. casei-OMP16-PEDVS strain. After blocking with 5\% skimmed milk, the collected samples were serially diluted in PBS, added in triplicate, and incubated at $37^{\circ} \mathrm{C}$ for $1 \mathrm{~h}$. Then, an HRP conjugated goat anti-mouse IgG or IgA antibody (Invitrogen, USA) was added to each well (1:5000) and incubated for $1 \mathrm{~h}$ at $37^{\circ} \mathrm{C}$. The polystyrene microtiter plates were washed 5 times during each step. At last, $100 \mu \mathrm{L}$ of TMB substrate (tetramethylbenzidine and $\mathrm{H}_{2} \mathrm{O}_{2}$ ) was added to each well and $50 \mu \mathrm{L}$ of stop solution was added after 10 mins. The OD values at $630 \mathrm{~nm}$ were measured using a multimode plate reader (EnVision).

\section{Virus neutralization assays}

The neutralizing antibody titers of PEDV in sera were examined according to the methods with some modifications $[25,26]$. Briefly, the murine serum was heat- 
Table 2 The immune protocol of BALB/C mice

\begin{tabular}{lllr}
\hline Grouping & Characteristics & Immune methods & Immunizing dose \\
\hline L. casei-OMP16-PEDVS & Recombinant strain L. casei contains pVE5523-OMP16-PEDVS vector & PO & $200 \mu \mathrm{L}$ \\
BL21-PEDVS & pET28a-PEDVS protein & $\mathrm{IM}$ & $200 \mu \mathrm{g}$ \\
BL21-OMP16-PEDVS & pET28a-OMP16-PEDVS protein & $\mathrm{IM}$ & $200 \mu \mathrm{\mu g}$ \\
BL21-OMP16-PEDVS-F & pET28a-OMP16-PEDVS protein with Freund's complete adjuvant & $\mathrm{IM}$ & $200 \mu \mathrm{g}$ \\
PBS & Negative control & $\mathrm{IM}$ & $200 \mu \mathrm{L}$ \\
\hline
\end{tabular}

inactivated $\left(56^{\circ} \mathrm{C}\right.$ for $\left.30 \mathrm{~min}\right)$ and then serially two-fold diluted in 96 well plates (Corning, USA) with triplicates of each sample. Then, an equal volume of $200 \mathrm{TCID}_{50} /$ $50 \mu \mathrm{L}$ PEDV strains were added to 96 well plates and incubated for $1 \mathrm{~h}$ at $37^{\circ} \mathrm{C}$. The mixture was added to new 96 well plates coated with Vero cell monolayers and incubated for $1 \mathrm{~h}$ at $37^{\circ} \mathrm{C}$. Cells were then washed and incubated in the maintenance medium at $37^{\circ} \mathrm{C}$ in $5 \%$ $\mathrm{CO}_{2}$. After 2 days, the cytopathic effect (CPE) was observed using an inverted microscope and the neutralizing concentration was defined as the lowest concentration of antibodies in the serum.

\section{Cytokine detection}

To detect the secretion of cytokines, supernatants were obtained from the laboratory mice $(0,14$, and 28 days). Levels of secreted IL-4, IL-10, and IFN- $\gamma$ were determined using commercial ELISA kits (Elabscience Biotechnology Co., Ltd., Wuhan) according to the manufacturer's recommendations, respectively. Cytokine was quantified from the different standard curves prepared from standard reagents provided by the kits respectively and optical density (OD) value was detected at $450 \mathrm{~nm}$ from each plate using a multimode plate reader (EnVision) [23, 27].

\section{Statistical analysis}

All data were obtained from at least three independent experiments, and results were presented as the means \pm standard deviation (SD). The statistical analysis was performed using two-tailed $t$-tests and one-way analysis in Graph Pad Prism 7.0 (GraphPad Software Inc., USA). The significant difference was defined as $* p<0.05$, and the various degrees of significant difference were designated as *: $p<0.01$, :**: $p<0.001$, respectively.

\section{Results}

\section{The verification of recombinant Lactobacillus casei and BL21 strains}

To verify the constructed recombinant plasmids, the recombinant expression plasmids pET28a-PEDVS, pET28a-OMP16-PEDVS, and pVE5523-OMP16-PEDVS were digested using NcoI/XhoI restriction enzymes, and the enzyme digestion results were shown in Fig. 1. The sizes of target bands in electrophoretograms were the same as the expected results and sequencing results indicated that the recombinant expression plasmids exhibited no mutation. These results indicated the successful construction of pET28a-PEDVS, pET28aOMP16-PEDVS, and pVE5523-OMP16-PEDVS recombinant plasmids.

The results of western blot showed that the recombinant proteins pET28a-OMP16-PEDVS and pET28a-PEDVS were expressed in the supernatant of BL21-pET28aOMP16-PEDVS and BL21-pET28a-PEDVS strains, respectively. The pVE5523-OMP16-PEDVS protein was also verified in the cultural supernatant of L. casei-pVE5523OMP16-PEDVS strain. The specific bands from Fig. 2 showed that the recombinant proteins pET28a-OMP16PEDVS, pVE5523-OMP16-PEDVS, and pET28a-PEDVS were all harvested successfully (Fig. 2).

The IgG antibody levels in serum of mice immunized with candidate vaccines

To evaluate the specific immunogenicity of generated vaccine candidates, BALB/c mice were selected and divided into 5 groups. Then, the levels of IgG in the serum and IgA in the feces were measured with commercial ELISA kits. The results revealed that there were no substantial differences for IgG levels among the vaccinated groups and almost no IgG antibody was found in all mice before immunization. However, substantial differences were subsequently found after the first vaccination and IgG antibody levels in serum of 28 days were obviously higher than that of in 14 days. Among 5 group mice, the mice immunized with L. casei-OMP16PEDVS and BL21-OMP16-PEDVS-F showed similar and highest immunogenicity. Therefore, L. casei-OMP16PEDVS and BL21-OMP16-PEDVS-F could produce highest immunogenicity, followed by BL21-OMP16PEDVS and BL21-PEDVS (Fig. 3).

\section{The IgA antibody levels in feces of mice immunized with candidate vaccines}

To evaluate the specific immunogenicity of generated vaccine candidates, the levels of IgA antibody in feces of mice were also evaluated. The results showed that there was no special anti-PEDVS IgA antibody existed before immunization. However, large amounts of IgA antibody in feces of L. casei-OMP16-PEDVS immunized mice 

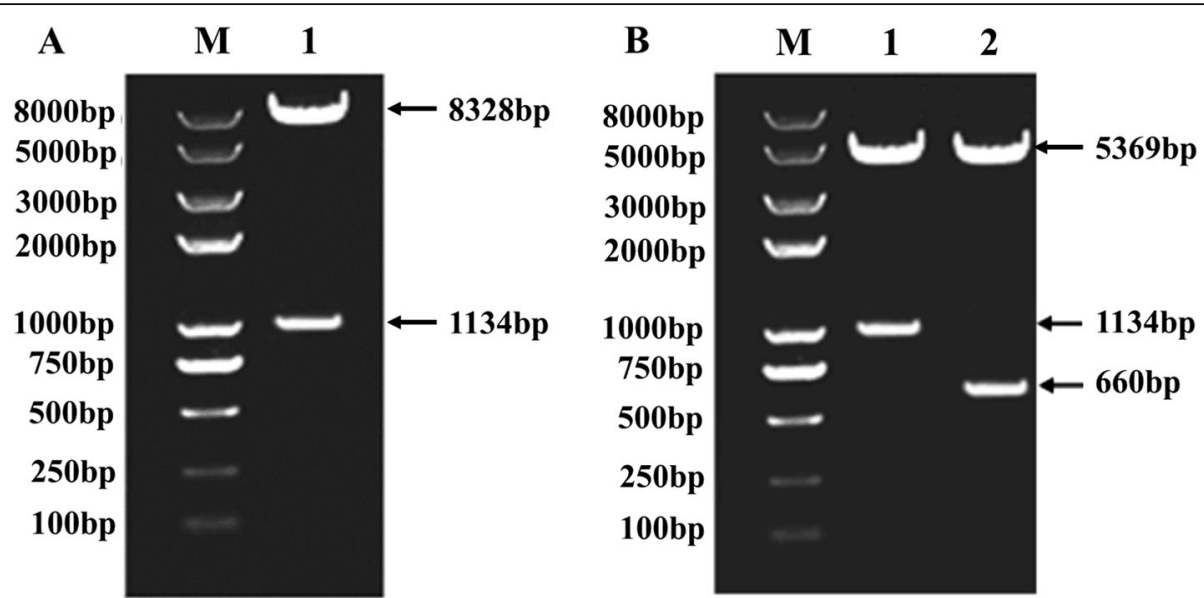

Fig. 1 The enzyme digestion results of recombinant plasmids. A: The enzyme digestion results of the pVE5523-OMP16-PEDVS plasmid. M: D8000 DNA Ladder Marker; 1: pVE5523-OMP16-PEDVS plasmid. B: The enzyme digestion results of pet28a-OMP16-PEDVS and pET28a-PEDVS plasmid. M: D8000 DNA Ladder Marker; 1: pet28a-OMP16-PEDVS plasmid; 2: pET28a-PEDVS plasmid

were detected and it was obviously higher than that of in BL21-OMP16-PEDVS-F, BL21-OMP16-PEDVS, and BL21-PEDVS group mice at 14 days after immunization. At 28 days after immunization, the IgA antibody levels of L. casei-OMP16-PEDVS immunized mice reached its highest maximum. Meanwhile, the IgA antibody levels in the other three groups did not present an obvious increase. Therefore, the candidate vaccine L. caseiOMP16-PEDVS could stimulate higher levels of antibody in immunized mice compared with BL21OMP16-PEDVS-F, BL21-OMP16-PEDVS, and BL21PEDVS immunized mice (Fig. 4).

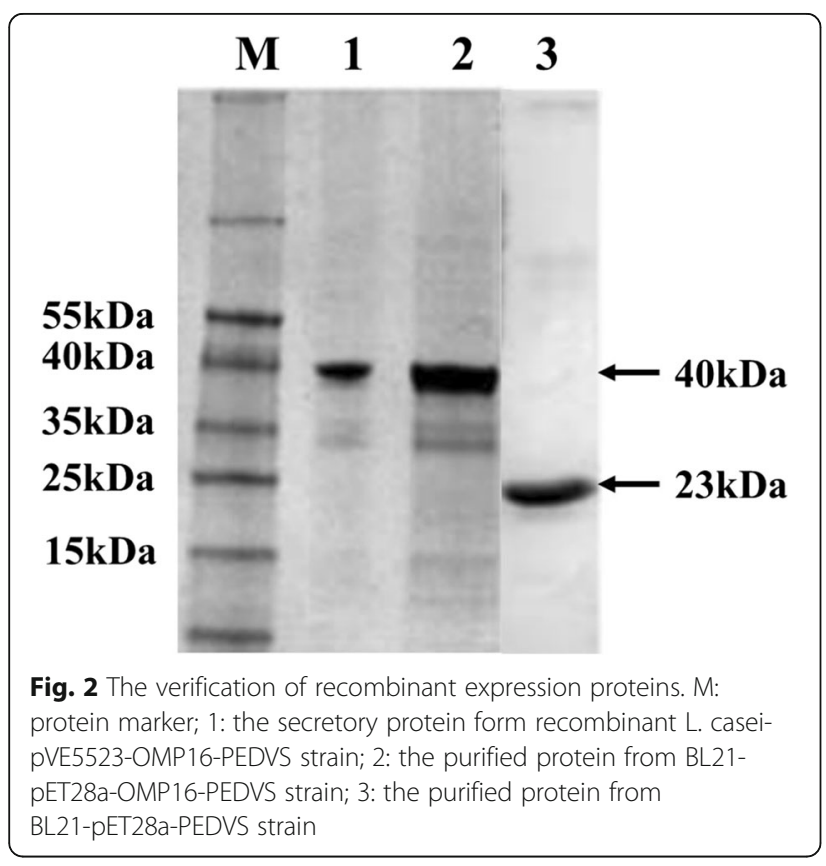

The neutralizing antibody levels of serum in immunized mice

To evaluate the protective effect of candidate vaccines of $\mathrm{L}$. casei-OMP16-PEDVS, BL21-OMP16-PEDVS-F, BL21OMP16-PEDVS, and BL21-PEDVS in mice, the neutralizing antibody levels were measured. Results showed that no neutralizing antibody was detected before immunization. Neutralizing antibody was detected at 14days after immunization and it increased at 14 days after booster immunization. The antibody response in mice that received L. casei-OMP16-PEDVS possessed a stronger anti-PEDV neutralizing activity than that in mice orally administered with BL21-OMP16-PEDVS-F, BL21-OMP16-PEDVS, and BL21-PEDVS. Therefore, the candidate vaccine $\mathrm{L}$. caseiOMP16-PEDVS could stimulate highest neutralizing antibody level, followed by BL21-OMP16-PEDVS-F, BL21OMP16-PEDVS, and BL21-PEDVS (Fig. 5).

\section{Cytokine levels}

To compare the cellular immune response level of L. casei-OMP16-PEDVS, BL21-OMP16-PEDVS-F, BL21OMP16-PEDVS, and BL21-PEDVS immunized mice, IL4 , IL-10, and IFN- $\gamma$ were determined, respectively. The results showed that the levels of cytokines IL-4, IL-10, and IFN- $\gamma$ in the sera of mice were all very low and have no significant difference before immunization. Whereas, similar changes were observed in the results of IL-4, IL10 , and IFN- $\gamma$. At 14 days after immunization, the level of IL-4, IL-10, and IFN- $\gamma$ in L. casei-OMP16-PEDVS immunized mice were higher than BL21-OMP16-PEDVSF, BL21-OMP16-PEDVS, and BL21-PEDVS immunized mice. At 14 days after the booster immunization, a higher IL-4, IL-10, and IFN- $\gamma$ level in L. casei-OMP16PEDVS immunized mice were detected compared with that of in other three groups. Therefore, the candidate 


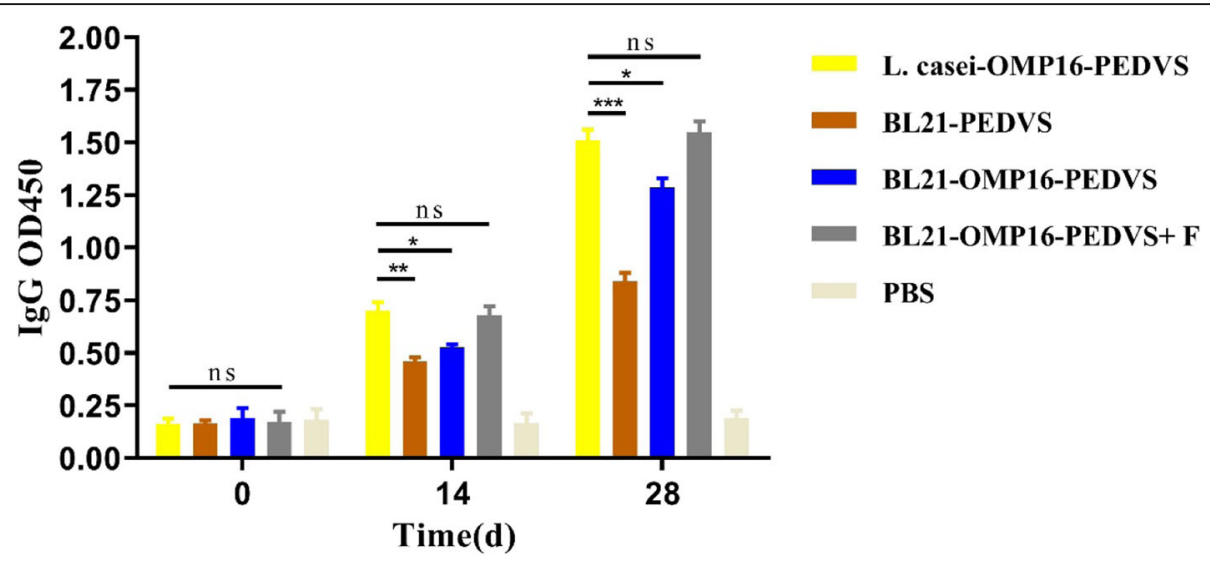

Fig. 3 The $\lg G$ antibody levels of candidate vaccines in the serum of immunized mice. Serum was collected on days 0,14 , and 28 days before or after immunization and examined via commercial ELISA kits and measured at an absorbance of $450 \mathrm{~nm}$. Bars represent the mean \pm standard deviation of three independent experiments. ${ }^{*} p<0.05$, ${ }^{* *} p<0.01$, and ${ }^{* * *} p<0.0001$ represent increasing degrees of significant differences, respectively, and ns means no significant difference

vaccine L. casei-OMP16-PEDVS could stimulate highest IL-4, IL-10, and IFN- $\gamma$ level, followed by BL21-OMP16PEDVS-F, BL21-OMP16-PEDVS, and BL21-PEDVS (Fig. 6).

\section{Discussion}

Since a large-scale outbreak of PED that caused by PEDV variants occurred in October 2010, which has resulted in tremendous economic losses in China and all around the world [1]. However, traditional vaccines are all designed based on CV777 classical strain which cannot supply sufficient protection to PEDV variant [4]. To control the spread of PEDV and reduce the economic losses, novel vaccines of PEDV variant strains are also designed. At present, PEDV inactivated vaccine and attenuated vaccine of PEDV variant strains are all approved by Chinese government and there are all exhibiting promising prospects in controlling PED. But defects are also existed in the two kinds of novel vaccines. PEDV infects swine through the digestive tract and has intestinal tissue tropism. Therefore, Mucosal immunity is more effective than systemic immunity in preventing PEDV entry into intestinal epithelial cells, and vaccines must provide mucosal protection effectively in the intestinal tract. So, In this study, we construct a new kind of vaccine which can stimulate stronger anti-PEDV-specific IgG and sIgA antibodies.

Lactobacillus casei has potential immune-modulatory properties as a vaccine delivery vehicle and the expression of bioactive compounds on the cell wall of this

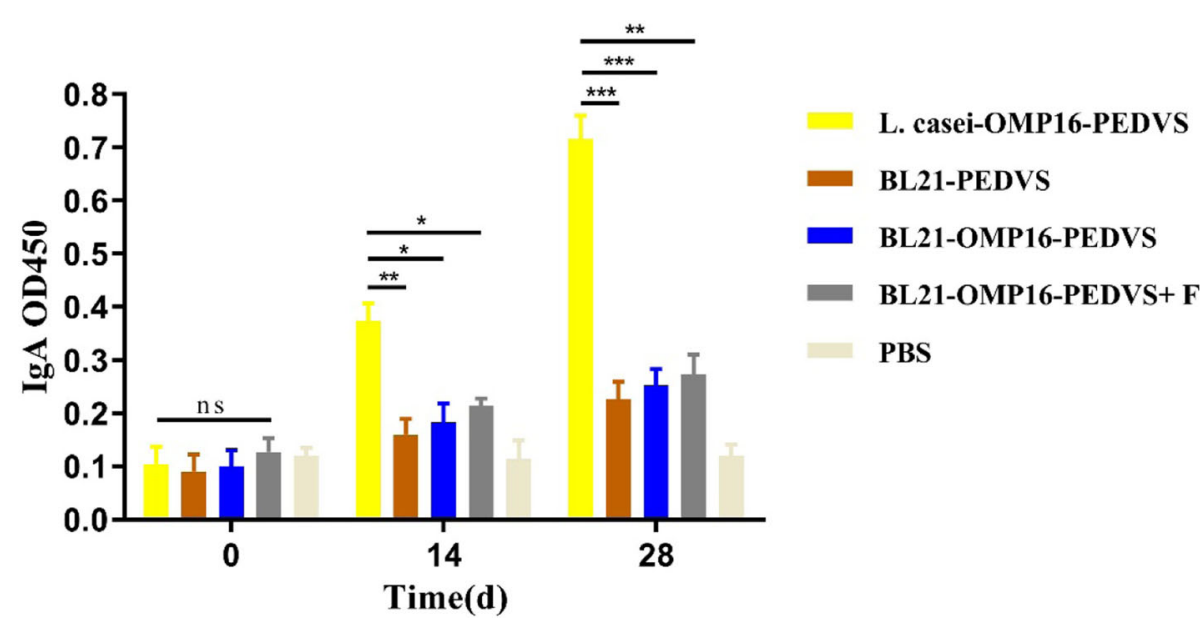

Fig. 4 The IgA antibody levels of candidate vaccines in feces of immunized mice. Feces were collected on day 0, 14, and 28 days before or after immunization and examined via commercial ELISA kits and measured at an absorbance of $450 \mathrm{~nm}$. Bars represent the mean \pm standard deviation of three independent experiments. ${ }^{*} p<0.05,{ }^{* *} p<0.01$, and ${ }^{* * *} p<0.0001$ represent increasing degrees of significant differences, respectively, and ns means no significant difference 


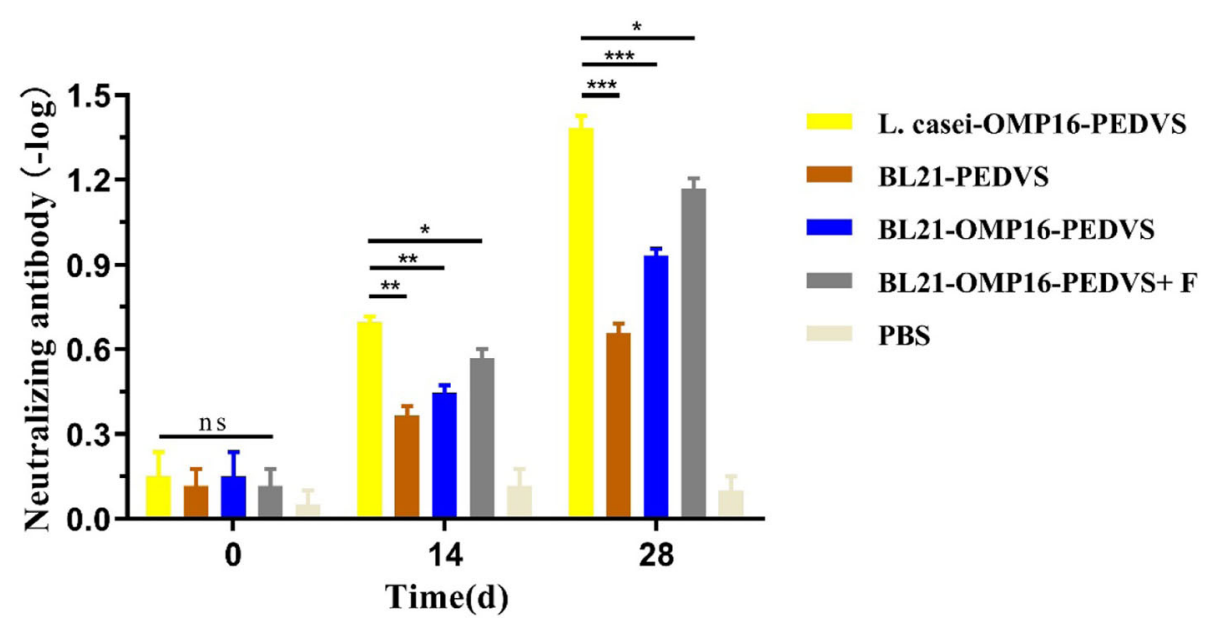

Fig. 5 The neutralizing antibody levels of candidate vaccines in the serum of immunized mice. Serum was collected on days 0,14 , and 28 days before or after immunization and examined via neutralization test. Bars represent the mean \pm standard deviation of three independent experiments. ${ }^{*} p<0.05,{ }^{* *} p<0.01$, and ${ }^{* * *} p<0.0001$ represent increasing degrees of significant differences, respectively, and ns means no significant difference

bacterium can stimulate appropriate immune responses $[28,29]$. It is widely used for expressing several heterologous antigens of human papillomavirus, Streptococcus pneumonia, and Escherichia coli as vaccines in animal models, which all showed excellent immunogenicity [79]. Compared with the inactivated vaccine and attenuated vaccine, the Lactobacillus casei vector vaccine can also stimulate higher IgA level and cellular immune response. Studies have shown that IgA's first line of defense in the intestine would be better than IgG in protecting piglets from PEDV infection [10, 30]. Therefore, it is promising to develop a kind of Lactobacillus casei vector vaccine of PED.

Based on the reports, the S protein of PEDV can be divided into S1 (1-735 amino acid) and S2 (736-last amino acid) domains [13], and $\mathrm{S} 1$ protein includes the receptor-binding region and the main neutralizing epitopes [14]. The core neutralizing epitope (COE) can induce strong neutralizing antibodies against PEDV [31, 32]. Combining with the antigenicity analysis, a partial sequence of the S1 gene (1477-2124 bp) was selected to construct the recombinant plasmid. The selected small fragment was proved that not only had good immunogenicity but also contributed to the secreted expression of Lactobacillus casei. On the other hand, Pasquevich found that Brucella abortus outer membrane protein 16 could activate dendritic cells in vivo, induces a th1 immune response, and was a promising self-adjuvanting vaccine against systemic and oral acquired brucellosis [15]. Similar research that unlipidated outer membrane protein omp16 from Brucella spp. as nasal adjuvant could induce a th1 immune response and modulates the th2 allergic response to cow's milk proteins was also proved [16]. Therefore, a partial sequence of the omp16 gene was chosen to construct recombinant plasmid to enhance to immune function in our study.

To know whether the novel Lactobacillus casei recombinant vaccine could induce humoral immune responses, the IgG, IgA, and neutralizing antibody levels were measured. The IgG antibody level of L. casei-OMP16PEDVS recombinant vaccine immunized mice had no significant difference with BL21-OMP16-PEDVS-F recombinant vaccine immunized mice. But the IgA and neutralizing antibody levels were obviously higher than that of in BL21-OMP16-PEDVS-F, BL21-OMP16-PEDV $S$, and BL21-PEDVS recombinant vaccine immunized mice. The results showed that L. casei-OMP16-PEDVS could induce stronger humoral immune responses, especially IgA antibody level. Studies have shown that IgA was the first line of defense in the intestine and would be better than IgG in protecting piglets from PEDV infection [30]. The research also verified the result that $L$. casei-OMP16-PEDVS recombinant vaccine could supply better immunological protection to PEDV.

To explore the type of immune response induced by recombinant L. casei-OMP16-PEDVS, the levels of IL-4, IL-10, and IFN- $\gamma$ were detected to evaluate the activity of $\mathrm{T}$ lymphocytes. Based on the report, IFN- $\gamma$ plays an important role in cellular immune response caused when pathogens invade the body, IL-4 plays an important role in the humoral immune response and promoting immune tolerance and mucosal immunity [33], IL-10 plays essential roles in fighting against mucosal microbial infection and maintaining mucosal barrier integrity within the intestine [34]. Meanwhile, results of cytokine detection showed that the mice immunized with $\mathrm{L}$. casei-OMP16-PEDVS recombinant vaccine could induce stronger expression of IL-4, IL-10, and IFN- $\gamma$, which 

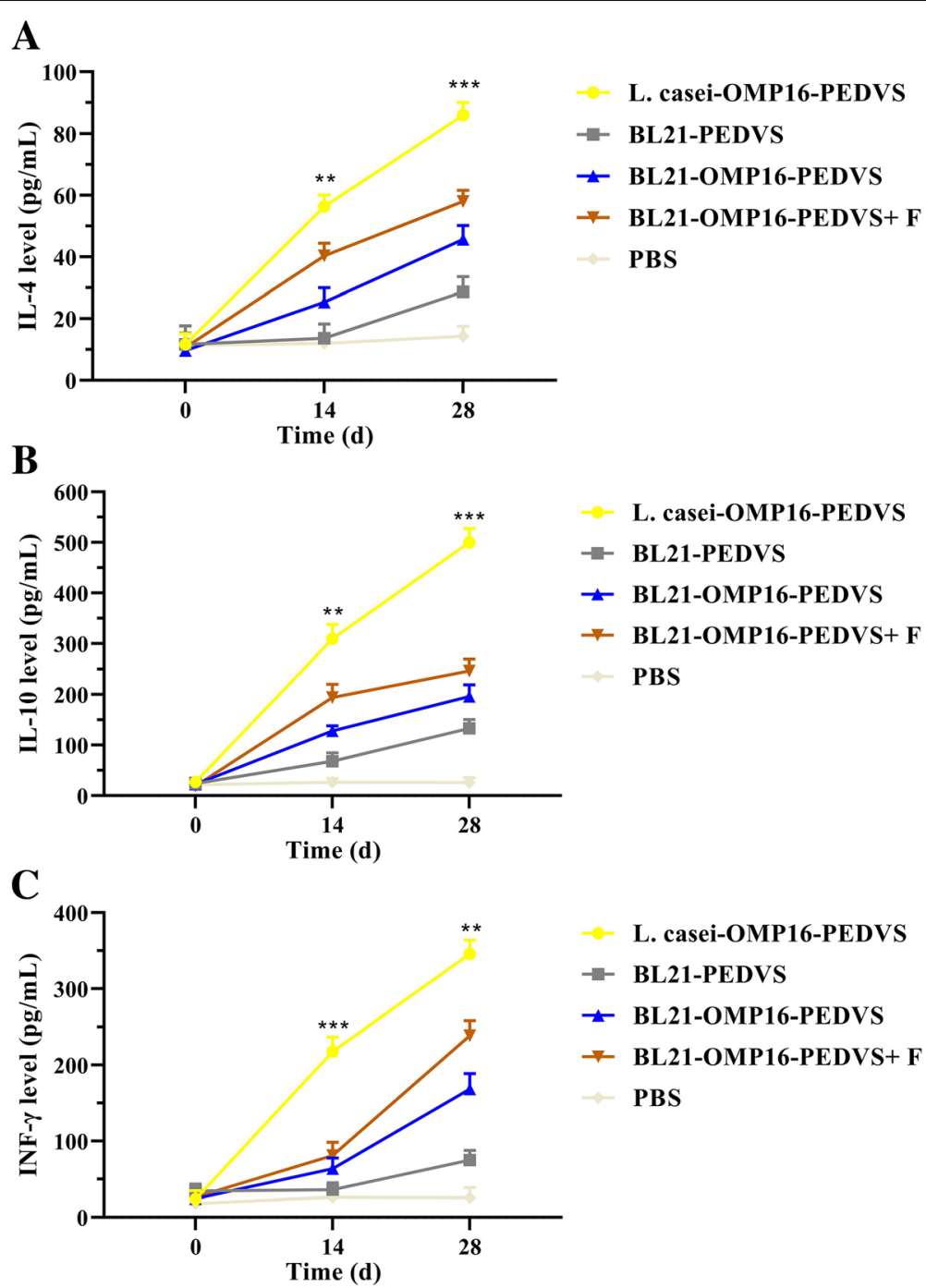

Fig. 6 Detection of cytokine levels from the serum of immunized mice. Serum was collected on days 0, 14, and 28 days before or after immunization and examined via commercial ELISA kits. The absorbance value was measured at an absorbance of $450 \mathrm{~nm}$ for IL-4 (a), IL-10 (b), and IFN $-\gamma(\mathbf{c})$, respectively. Bars represent the mean \pm standard deviation of three independent experiments. ${ }^{*} p<0.05,{ }^{* *} p<0.01$, and ${ }^{* * * *} p<$ 0.0001 represent increasing degrees of significant differences, respectively

supported the results that L. casei-OMP16-PEDVS recombinant vaccine could induce stronger humoral immune response, IgA antibody level, and cellular immune response, respectively.

\section{Conclusion}

In summary, L. casei-OMP16-PEDVS, BL21-OMP16PEDVS-F, BL21-OMP16-PEDVS, and BL21-PEDVS candidate vaccines were constructed in this study. Meanwhile, the humoral immune response and cellular immune response levels of these candidate vaccines in mice were evaluated. The results showed that the mice immunized with L. casei-OMP16-PEDVS could produce higher levels of IgG, IgA, neutralizing antibody, IL-4, IL10 , and INF- $\gamma$ compared with the mice immunized with
BL21-OMP16-PEDVS-F, BL21-OMP16-PEDVS, and BL21-PEDVS. Therefore, the recombinant L. caseiOMP16-PEDVS candidate vaccine may establish the ground for the development of a safe, effective, and convenient recombinant mucosal vaccine for prophylaxis of PEDV infection.

\section{Supplementary Information}

The online version contains supplementary material available at https://doi. org/10.1186/s12917-021-02885-y.

\section{Additional file 1.}

Statement

The study was carried out in compliance with the arrive guidelines. 


\section{Authors' contributions}

Conceptualization, Xiaowen Li and Jing Ren; Data curation, Xiaowen Li and Bingzhou Zhang; Formal analysis, Bingzhou Zhang and Dasheng Zhang; Funding acquisition, Sidang Liu and Jing Ren; Investigation, Xiaowen Li and Dasheng Zhang; Methodology, Xiaowen Li, Bingzhou Zhang and Dasheng Zhang; Project administration, Sidang Liu; Resources, Dasheng Zhang, Sidang Liu and Jing Ren; Software, Bingzhou Zhang; Supervision, Sidang Liu and Jing Ren; Validation, Xiaowen Li and Jing Ren; Visualization, Xiaowen Li; Writing - original draft, Bingzhou Zhang; Writing - review \& editing, Bingzhou Zhang. The authors read and approved the final manuscript.

\section{Funding}

This research was supported by New Hope Group Science and Technology Leading 100-person Program Fund (No. NH201806), National Natural Science Foundation Of China (NSFC) (No. 31701424), and Shandong Provincial Key Laboratory Open Fund (No. SD2019BP002).

\section{Declarations}

\section{Ethics approval and consent to participate}

In the present study, all the experimental methodology on animals were performed in strict accordance with the guideline for experimental animals approved by the Ethics Committee of the Faculty of Veterinary Medicine, Shandong Agricultural University, China. All procedures followed the instruction of the care and use of laboratory animals provided by Shandong provincial public service facilities. The authors state their agreement to publication ethics. This study does not include any experiment performed on human participants.

\section{Consent for publication}

The authors have agreed to publish all the identifiable images and identifiers in the paper.

Availability of data and materials: All data generated or analysed during this study are included in this published article and its supplementary information files.

\section{Competing interests}

The authors have declared that no competing financial interests exist.

\section{Author details}

'Swine Research Institute of New Hope Group, Tai'an, China. ${ }^{2}$ Shandong Swine Herd Health Big Data and Intelligent Monitoring Engineering Laboratory, Tai'an, China. ${ }^{3}$ College of Animal Science and Technology, Shandong Agricultural University, Tai'an, China. ${ }^{4}$ Chang Sha Axybio Bio-tech Co., Ltd, Changsha, China. ${ }^{5}$ Shandong Provincial Key Laboratory of Biophysics, Institute of Biophysics, Dezhou University, Dezhou 253023, China.

\section{Received: 21 December 2020 Accepted: 15 April 2021}

\section{Published online: 04 May 2021}

\section{References}

1. Wang D, Fang L, S X. Porcine epidemic diarrhea in China. Virus Res. 2016; 226:7-13.

2. Sueyoshi M, Tsuda T, Yamazaki K, Yoshida K, Nakazawa M, Sato K, et al. An immunohistochemical investigation of porcine epidemic diarrhoea. J Comp Pathol. 1995;113(1):59-67.

3. Sun RQ, Cai RJ, Chen YQ, Liang PS, Song CXJEID. Outbreak of porcine epidemic diarrhea in suckling piglets, China. Emerg Infect Dis. 2012; 18(1):161-3.

4. Tong YE, Feng L, Li W. Development of bi-combined attenuated vaccine against transmissible gastroenteritis virus and porcine epidemic diarrhea virus. Chin J Prev Vet Med. 1999;21(6):35-9.

5. Pouwels PH, Leer RJ, Boersma WJ. The potential of Lactobacillus as a carrier for oral immunization: development and preliminary characterization of vector systems for targeted delivery of antigens. J Biotechnol. 1996:44(1-3):183.

6. Tsai YT, Cheng PC, Pan TM. Biotechnology: The immunomodulatory effects of lactic acid bacteria for improving immune functions and benefits. Appl Microbiol Biotechnol. 2012;96(4):853-62.

7. Adachi K, Kawana K, Yokoyama T, Fujii T, Tomio A, Miura S, et al. Oral immunization with a Lactobacillus casei vaccine expressing human papillomavirus (HPV) type 16 E7 is an effective strategy to induce mucosal cytotoxic lymphocytes against HPV16 E7. Vaccine. 2010;28(16):2810-7.

8. Campos IB, Darrieux M, Ferreira DM, Miyaji EN, Silva DA, Arêas APM, et al. Nasal immunization of mice with Lactobacillus casei expressing the Pneumococcal Surface Protein A: induction of antibodies, complement deposition and partial protection against Streptococcus pneumoniae challenge. Microbes Infect. 2008;10(5):481-8.

9. Wen LJ, Hou XL, Wang GH, Yu LY, Wei XM, Liu JK, et al. Immunization with recombinant Lactobacillus casei strains producing K99, K88 fimbrial protein protects mice against enterotoxigenic Escherichia coli. Vaccine. 2012;30(22): 3339-49.

10. Guo M, Yi S, Guo Y, Zhang S, Niu J, Wang K, et al. Construction of a recombinant lactococcus lactis strain expressing a variant porcine epidemic diarrhea virus $\mathrm{S} 1$ gene and its immunogenicity analysis in mice. Viral Immunol. 2019;32(3):144-50.

11. Wang $X$, Wang $L$, Zheng D. Oral immunization with a Lactobacillus caseibased anti-porcine epidemic diarrhea virus (PEDV) vaccine expressing microfold cell-targeting peptide Col fused with the COE antigen of PEDV. J Appl Microbiol. 2017;124(2):368-78.

12. Duarte M, Tobler K, Bridgen A, Rasschaert D, Ackermann M, H L. Sequence analysis of the porcine epidemic diarrhea virus genome between the nucleocapsid and spike protein genes reveals a polymorphic ORF. Virology. 1994;198(2):466

13. Lee DK, Park CK, Kim SH, Lee C. Heterogeneity in spike protein genes of porcine epidemic diarrhea viruses isolated in Korea. Virus Res. 2010;149(2): 175-82.

14. Sun DB, Feng L, Shi HY, Chen JF, Liu SW, Chen HY, et al. Spike protein region (aa 636789) of porcine epidemic diarrhea virus is essential for induction of neutralizing antibodies. Acta Virol. 2007;51(3):149-56.

15. Pasquevich KA, Garcia Samartino C, Coria LM, Estein SM, Zwerdling A, Ibanez $A E$, et al. The protein moiety of Brucella abortus outer membrane protein 16 is a new bacterial pathogen-associated molecular pattern that activates dendritic cells in vivo, induces a Th1 immune response, and is a promising self-adjuvanting vaccine against systemic and oral acquired brucellosis. J Immunol. 2010;184(9):5200.

16. Ibanez AE, Smaldini P, Coria LM, Delpino MV, Pacífico LGG, Oliveira SC, et al. Unlipidated outer membrane protein Omp16 (U-Omp16) from Brucella spp. as nasal adjuvant induces a Th1 immune response and modulates the Th2 allergic response to cow's milk proteins. PLoS One. 2013;8(7):e69438.

17. Pasquevich KA, Estein SM, Samartino CG, Zwerdling A, Coria LM, Barrionuevo P, et al. Immunity: immunization with recombinant Brucella species outer membrane protein Omp16 or Omp19 in adjuvant induces specific CD4+ and CD8+ T cells as well as systemic and oral protection against Brucella abortus infection. Infect Immun. 2009;77(1):436-45.

18. Ma S, Wang L, Huang $X$, Wang $X$, Chen S, Shi W, et al. Oral recombinant Lactobacillus vaccine targeting the intestinal microfold cells and dendritic cells for delivering the core neutralizing epitope of porcine epidemic diarrhea virus. Microb Cell Factories. 2018;17(1):20.

19. Chu S, Zhang D, Wang D, Zhi Y, Zhou P. Heterologous expression and biochemical characterization of assimilatory nitrate and nitrite reductase reveals adaption and potential of Bacillus megaterium NCT-2 in secondary salinization soil. Int J Biol Macromol. 2017;101:1019.

20. Guo N, Zhang B, Hu H, Ye S, Chen F, Li Z, et al. Caerin1.1 suppresses the growth of porcine epidemic diarrhea virus in vitro via direct binding to the virus. Viruses. 2018;10:9.

21. Chen Z, Lin J, Ma C, Zhao S, She Q, Liang Y. Biotechnology: characterization of pMC11, a plasmid with dual origins of replication isolated from Lactobacillus casei MCJ and construction of shuttle vectors with each replicon. Appl Microbiol Biotechnol. 2014;98(13):5977.

22. Xiaona W, Li W, Xuewei H, Sunting M, Meiling Y, Wen S, et al. Oral Delivery of Probiotics Expressing Dendritic Cell-Targeting Peptide Fused with Porcine Epidemic Diarrhea Virus COE Antigen: A Promising Vaccine Strategy against PEDV. Viruses. 2017:9(11):312.

23. Bhuyan AA, Memon AM, Bhuiyan AA, Zhonghua L, Zhang B, Ye S, et al. The construction of recombinant Lactobacillus casei expressing BVDV E2 protein and its immune response in mice. J Biotechnol. 2018;270:51-60

24. Noi NV, Chung YCJB, Equipment B. Optimization of expression and purification of recombinant S1 domain of the porcine epidemic diarrhea virus spike (PEDV- S1) protein in Escherichia coli. Biotechnol Biotechnol Equip. 2017;31(2):1-11. 
25. Li C, Li W, Esesarte E, Guo H, Elzen P, Aarts E, et al. Cell attachment domains of the porcine epidemic diarrhea virus spike protein are key targets of neutralizing antibodies. J Virol. 2017;91(12):1-16.

26. Wen Z, Xu Z, Zhou Q, Li W, Wu Y, Du Y, et al. Oral administration of coated PEDV-loaded microspheres elicited PEDV-specific immunity in weaned piglets. Vaccine. 2019;09(014):161-6.

27. Gao Q, Zhao S, Qin T, Yin Y, Yang Q. Effects of porcine epidemic diarrhea virus on porcine monocyte-derived dendritic cells and intestinal dendritic cells. Vet Microbiol. 2016;106:149-58.

28. Bonet MEB, Chaves AS, Mesón O. Immunomodulatory and antiinflammatory activity induced by oral administration of a probiotic strain of lactobacillus casei. Inflammation. 2006;4(1):31-41.

29. Grangette C, Müller-Alouf H, Geoffroy MC, Goudercourt D, Turneer M, Mercenier A. Protection against tetanus toxin after intragastric administration of two recombinant lactic acid bacteria: impact of strain viability and in vivo persistence. Vaccine. 2002;20(27-28):3304-9.

30. Song DS, Oh JS, Kang BK, Yang JS, Moon HJ, Yoo HS, et al. Oral efficacy of Vero cell attenuated porcine epidemic diarrhea virus DR13 strain. Res Vet Sci. 2007:82(1):134-40.

31. Makadiya N, Brownlie R, Jan V, Berube N, Allan B, Gerdts V, et al. S1 domain of the porcine epidemic diarrhea virus spike protein as a vaccine antigen. Virol J. 2016:13:1

32. Chang SH, Bae JL, Kang TJ, Ju K, Chung GH, Lim CW, et al. Cells: Identification of the epitope region capable of inducing neutralizing antibodies against the porcine epidemic diarrhea virus. Mol Cell. 2002;14(2): 295-9.

33. Sumi T, Fukushima A, Fukuda K, Kumagai N, Nishida T, Yagita H, et al. Differential contributions of B7-1 and B7-2 to the development of murine experimental allergic conjunctivitis. Immunol Lett. 2007;108(1):62-7.

34. Xue M, Zhao J, Ying L, Fu F, Li L, Ma Y, et al. IL-22 suppresses the infection of porcine enteric coronaviruses and rotavirus by activating STAT3 signal pathway. Antivir Res. 2017;142:68-75.

\section{Publisher's Note}

Springer Nature remains neutral with regard to jurisdictional claims in published maps and institutional affiliations.

Ready to submit your research? Choose BMC and benefit from:

- fast, convenient online submission

- thorough peer review by experienced researchers in your field

- rapid publication on acceptance

- support for research data, including large and complex data types

- gold Open Access which fosters wider collaboration and increased citations

- maximum visibility for your research: over $100 \mathrm{M}$ website views per year

At $\mathrm{BMC}$, research is always in progress.

Learn more biomedcentral.com/submissions 